\title{
Flexible solid supercapacitor, based on reduction oxidized graphene polymerization (3,4-ethylenedioxythiophene)
}

\author{
Zhigang Liu ${ }^{l}$, Dongyun Sü ${ }^{2}$ Jie Ding ${ }^{l}$, and Jun $\mathrm{Ma}^{1,3, *}$ \\ ${ }^{1}$ School of Mechatronics \& Traffic Engineering, Nantong College of Science and Technology, Nantong, China \\ ${ }^{2}$ College of Mechanical Engineering, Nantong Vocational University, Nantong, China \\ ${ }^{3}$ Institute of Materials Engineering, Nanjing University, Nantong, China
}

\begin{abstract}
In this case, RGO (graphene reduced load) aggregates (3, 4-ethylenedioxythiophene) were used to prepare basic films by chemical deposition method. Optimize electrochemical deposition (3, 4-ethylene dioxide) and rGO voltage Windows and electrolytes. SEM observation confirmed that PEDOT/rGO did synthesize the necessary composites. Electrochemical tests showed that the electrochemical performance of PEDOT electrode was improved by adding rGO. The mixed PEDOT/rGO electrode has a high specific capacitance $\left(134 \mathrm{~F} / \mathrm{g}, 0.8 \mathrm{~mA} / \mathrm{cm}^{2}\right)$.
\end{abstract}

\section{Introduction}

Supercapacitor the power storage device was emerged as an alternative electrochemical capacitor, from the energy storage device/power [1-3], flexible energy storage highly developed superior performance devices can have the the requirement for a large of the next generation energy, which was applied in executive fields but not limited to roll, displays artificial electronic already and high power and high cyclic life [4-6].

In general, according to the energy storage mechanism supercapacitors can be divided into two categories. One is an electrochemical double layer capacitor (EDLC), which typically take a high power density carbon material as the accumulation of pure electrostatic charges in the double layer. The other is a Faraday pseudocapacitor with transition metal oxides/hydroxides or conductive polymers, whose high energy density is derived from the surface of electroactive substances or surface REDOX reactions [7]. Electrode materials are crucial to the performance of supercapacitors. However, each electrode material has its advantages and disadvantages. For example, carbon materials show long cycle stability and high power density, but they have low capacitance. Transition metal oxides exhibit higher energy density than carbon materials and better cycle life than conductive polymers, but they also have the disadvantage of poor electrical conductivity. Conductive polymers have high energy density, but they reveal the disadvantage of low cycle life due to swelling and contraction during anti-ion doping/de-doping [8]. Conductive polymers have been the focus of extensive research in the past few years. Conductive polymers, such as polyaniline (PANI), polymagnetic (PPy), poly (3, 4-ethylene-dioxyethyl
mercols)(PEDOT) and their derivatives, have been widely studied by many researchers as promising electroactive materials for supercapacitors. The molecular structure of PEDOT is shown in Fig.1. Among the conductive polymer families, polymercaptan and its derivatives, such as PEDOT, have attracted the most interest. ECPS electrode materials due to chemical stability and good thermal properties, high conductivity (up to $550 \mathrm{~s} / \mathrm{cm}$ ), fast switching,low toxicity, especially a wider potential window $(>2.0 \mathrm{~V})$.

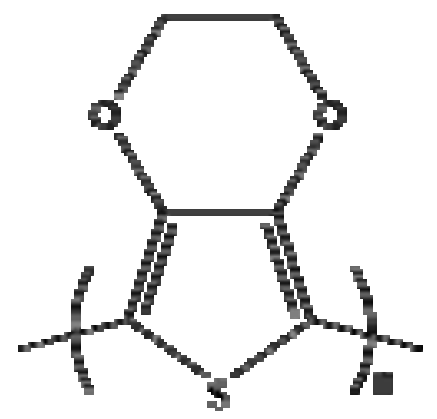

Fig. 1. Molecular structure of PEDOT[9].

RGO as an electrode material has been widely used in supercapacitors because of its high conductivity, small specific gravity, ideal specific surface area and good chemical stability [10]. Due to the hydrophilic properties of rGO and the ring between pep stacking layer and polymer the rGO / CPs composites have good capacitance properties, which also hinders the insulation properties of their charge storage properties, which is due to the structure of a large number of oxygencontaining functional groups [11]. RGO/PEDOT composites have been prepared by two-step electrochemical co-deposition [12] .

\footnotetext{
*Corresponding author: mjsdy@126.com
} 
In the study, the electrochemical behavior of (CV), constant current charging and discharging method (GCD) was studied and compared by cyclic volt-ampere method. Two same rGO/PEDOT composite electrodes were assembled into flexible solid state supercapacitors to verify the feasibility of the prepared composite electrode. It was found that the capacitance of the PEDOT/rGO composite electrode was as high as $134 \mathrm{~F} / \mathrm{g}$, and it had good rate performance.

\section{Experimental}

\subsection{Materials}

Carbon fiber cloth (HCP330: plain-weave, $165 \mathrm{~g} \mathrm{~m}-2$, no wet-proofing, $0.36 \mathrm{~mm}$ thickness) was purchased from Hesen Co., Ltd(Shanghai, China). Carbon cloth sheets with the size of $10 \mathrm{~mm} \times 20 \mathrm{~mm}$ were treated in $5 \%$ potassium permanganate for $60 \mathrm{~min}$, cleaned many times in alcohol and DI water, and dried at $70^{\circ} \mathrm{C}$ for $6 \mathrm{~h}$. The bearded CFC increased the surface area of the carbon fiber. Natural graphite powder (325 mesh) was purchased from Guangfu Research Institute (Tianjin, China). 3, 4-ethylenedioxythiophene (EDOT, Ourchem $\AA$, 99\%) and polyvinyl alcohol (PVA)-124 were purchased from Shanghai traditional Chinese Medicine holding Co., Ltd.

\subsection{Preparation of the electrode}

Graphite oxide was prepared by improved Hummers method with natural graphite powder as raw material, and graphene oxide was obtained by ultrasonic peeling. Deposited in aqueous solution containing $0.01 \mathrm{M}$ EDOT monomer and $3 \mathrm{mg} \mathrm{mL}^{-1}$ graphene oxide.

PEDOT-rGO composite thin films were prepared by electrochemical reduction and electrodeposition of REDOT. PEDOT-rGO was deposited on PEDOT for five periods. Then, PEDOT / CFC composite electrode was used for electrochemical reduction of graphene oxidation in ph8.0 graphene oxide solution (GNS). Finally, the PEDOT / RGO composite electrode was prepared by electrochemical polymerization of five cycles of PEDOT. Then the sandwich PEDOT-RGO electrode was dried at $70{ }^{\circ} \mathrm{C}$ for $6 \mathrm{~h}$.

\subsection{Characterizations}

The morphology of PEDOT-rGO composite electrode was studied by Japan JEOL scanning electron microscope (SEM, JSM-6510). All electrochemical behaviors include cyclic voltammograms (CV), constant current charge-discharge (CD) curve and electrochemical impedance spectroscopy (EIS) measurement using electrochemical workstation (CHI $660 \mathrm{E}$, Chenhua, China) at $1.0 \mathrm{~mol} / \mathrm{L}$ sulfuric acid aqueous solution and trielectrode system, saturated calomel electrode (SCE) as reference electrode and a large area $\mathrm{Pt}$ and reverse electrode foil. The electrochemical behavior was characterized between the potential of-0.4 0.6 V vs. SCE.

\section{Results and discussion}

\subsection{Morphology}

The SEM image of PEDOT/rGO composite was shown in Fig.2. The image shows the microstructure of twodimensional nanosheet connected with each other, and the curly sheet morphology of rGO is clearly observed, indicating that $\mathrm{rGO}$ plays a dominant role in the composite. It is worth noting that the curly sheet morphology of rGO disappears to a large extent, which is due to the fact that a large number of PEDOT coatings cover its morphology on rGO. The results show that the addition of rGO enhances the polymerization of PEDOT, which will help to strengthen the Faraday pseudocapacitance of the composite and promote the ion diffusion of electrolytes in the composite membrane.

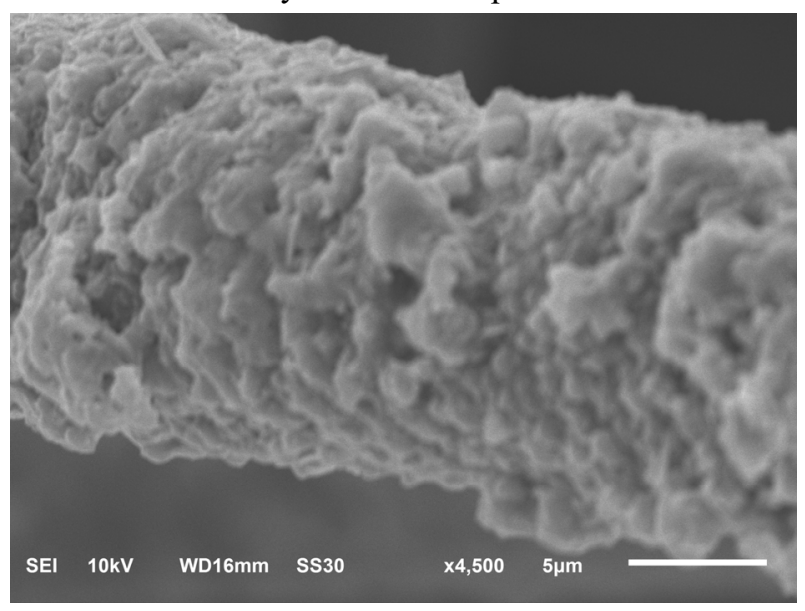

Fig. 2. SEM of rGO/PEDOT

\subsection{Electrochemical measurements}

In $1 \mathrm{M} \mathrm{H}_{2} \mathrm{SO}_{4}$ aqueous solution, the electrochemical behavior of the prepared PEDOT/rGO composite electrode was evaluated by $\mathrm{CV}$ measurement in a 5 $100 \mathrm{mv} / \mathrm{s}$ three-electrode system. As shown in Fig.3, the $\mathrm{CV}$ curve is approximately rectangular at $10 \mathrm{mv} / \mathrm{s}$, indicating that it has high capacitance and superior ion response performance. With the increase of scanning rate, the CV curve still maintains good symmetry, and a pair of wide redox peaks appear at $0.75 \mathrm{~V}$ and $0.05 \mathrm{~V}$, which is related to the anti-ion doping / anti-doping of PEDOT.

For the application of small electronic and fixed energy storage equipment, the area capacitance is a better index to measure the performance of supercapacitors compared with the weight specific capacitance. In addition, because the electrode materials are loaded on the substrate by electrodeposition, they are too light to be weighted accurately. 


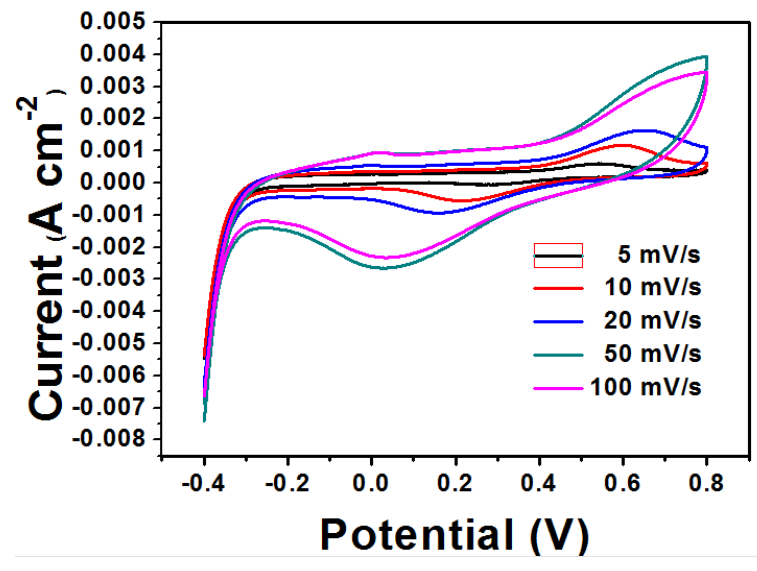

Fig. 3. Cyclic voltammogram curves of the PEDOT/rGO electrode at different scan rates $(5 \sim 100 \mathrm{mV} / \mathrm{s})$

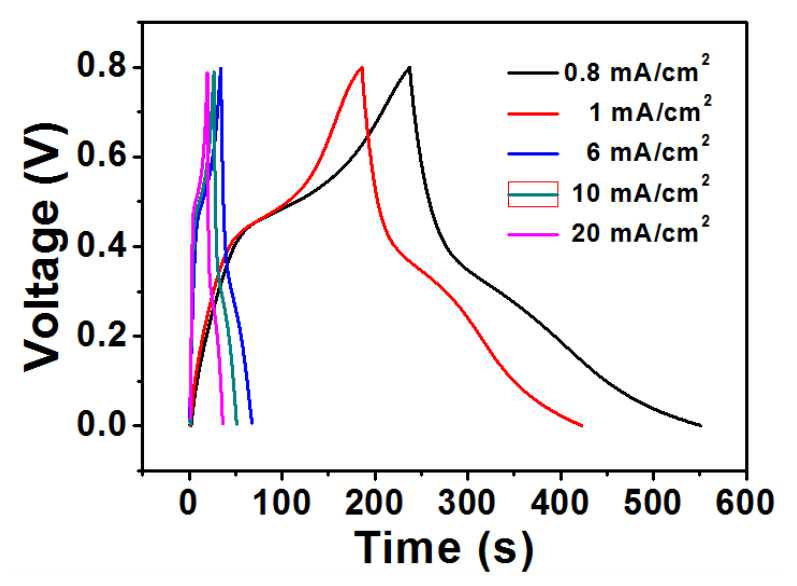

Fig. 4. Galvanostatic discharge profiles at various current densities $\left(2 \sim 20 \mathrm{~mA} / \mathrm{cm}^{2}\right)$

In order to further study the capacitive behavior of the composites, constant current charge and discharge measurements were carried out at $1.0 \mathrm{~A} / \mathrm{cm} 2$ current density, as shown in Fig. 4. In $1 \mathrm{~mol} / \mathrm{L} \mathrm{H} 2 \mathrm{SO} 4$, the $\mathrm{PEDOT} / \mathrm{rGO}$ electrode prepared with different concentrations of Aniline carries out constant current charge-discharge cycle between current density 0.8-20 $\mathrm{mA} / \mathrm{cm} 2$. It can be seen that the PEDOT/rGO value is quite high at all cyclic current densities, and the supercapacitance decreases with the increase of current density. With the increase of charge-discharge current density, the utilization rate of PEDOT decreases, which leads to the decrease of supercapacitor capacity. At 0.8 $\mathrm{mA} / \mathrm{cm}^{2}$, the capacitance value is as high as $134 \mathrm{~F} / \mathrm{g}$.

The Nyquist plot of PEDOT/rGO electrode displays an arc in the high frequency region, followed by a 45 degree line in the low frequency region (Fig. 5). The equivalent series resistance can be estimated from the $\mathrm{x}-$ intercept of the Nyquist plot to be about $4.5 \Omega$.

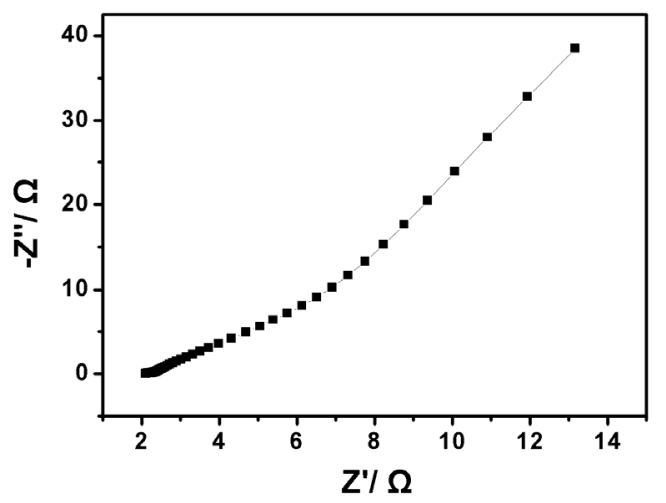

Fig. 5. Nyquist plot of PEDOT/rGO electrode

\section{Conclusion}

In summary, the composite electrode with hybrid structure of rGO supported PEDOT was prepared by simple electrochemical co-deposition. Electrochemical tests show that the synthesized PEDOT/rGO electrode has good capacitance, which is related to the optimum composition and specific microstructure of PEDOT/rGO electrode. A high flexible solid state supercapacitor based on composite materials has been developed, which has the characteristics of ultra-thin, lightweight and high flexibility, and has high specific capacitance. At 0.8 $\mathrm{mA} / \mathrm{cm} 2$, the capacitance value is as high as $134 \mathrm{~F} / \mathrm{g}$. Its superior performance and easy to manufacture show that the supercapacitor is a good choice for high flexibility and lightweight integrated electronic devices.

\section{Acknowledgments}

This work was financially supported by the Qing Lan Project of Jiangsu Province, the Natural Science Foundation of Jiangsu Province (BK20161289), Natural Science Foundation of Higher Education of Jiangsu Province (17KJB610009,18KJB43022), Six talent peaks project in Jiangsu Province (XCL-206), the College Students Innovation and Entrepreneurship Training Program of Jiangsu Province (201612684001Y), The enterprise practice for Higher Vocational Colleges Teachers of Jiangsu Province (2018QYSJ074), Innovation fund for colleges production, study and research of the education ministry's science and technology development center (2018A05027), 333 Talents Program of Jiangsu Province and Nantong Science and Technology Project (MS12018075, MS12017027-5, JC2018110).

\section{References}

1. F. Meng, Y. Ding, Adv. Mater. 23 (2011)

2. X. Li, X. Zang, Z. Li, X. Li, P. Li, P. Sun, X. Lee, R. Zhang, Z. Huang, K. Wang, D. Wu, F. Kang, H. Zhu, Adv. Funct. Mater. 23 (2013) 
3. L. Pan, G. Yu, D. Zhai, H. Lee, W. Zhao, N. Liu, H. Wang, B. Tee, Y. Shi, Y. Cui, Z. Bao, PNAS. 109 (2012)

4. J. Ma, S.C. Tang, J.A. Syed and X.K. Meng, RSC Adv. 6 (2016)

5. V. T. Le, H. Kim, A. Ghosh, J. Kim, J. Chang, Q.A. Vu, D.T. Pham, J.H. Lee, S.W. Kim, Y.H. Lee, ACS NANO. 7 (2013)

6. G. M. Wang, H.Y. Wang, X. H. Lu, Y. C. Ling, M. H. Yu, T. Zhai, Y. X. Tong, Y. Li, Adv. Mater. 26 (2014)

7. A. K. Mishra, S. Ramaprabhu, J. Phys. Chem. C.115 (2011)

8. T. Gueshi, K. Tokuda, H. Matsuda, Journal of Electroanalytical Chemistry and Interfacial Electrochemistry. 101 (1979)

9. G.A. Snook, G. Z. Chen, Journal of Electroanalytical Chemistry 612 (2008)

10. J. Wang, Y. Xu, X. Chen, X.F. Du, Journal of Power Sources 163 (2007)

11. B. Babakhani, D.G. Ivey, Electrochimica Acta 55 (2010)

12. J. Ma, S.C. Tang, J.A. Syed, D.Y. Su, X.K. Meng, Journal of Materials Science\& Technology, 34 (2018) 